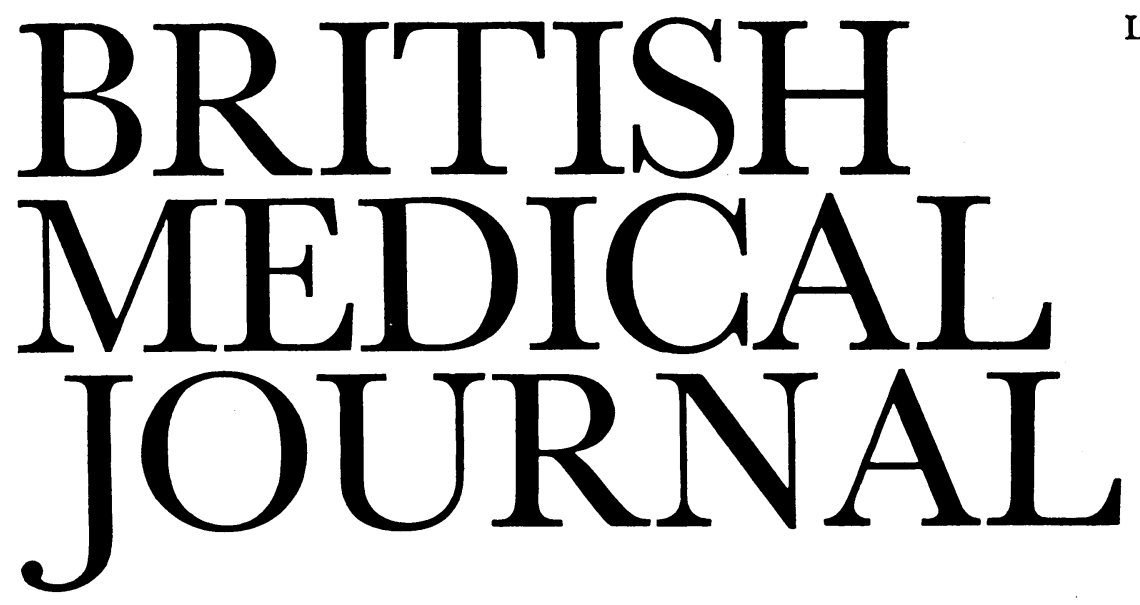

\title{
Environmental dangers for the patient with a pacemaker
}

Implantation of a pacemaker is a simple procedure carrying a negligible risk, and once the device is functioning well it may be expected to do so for upwards of five years. ${ }^{1}$ The patient with a pacemaker lives, however, in a potentially hostile environment in which electrical signals from an ever-increasing variety of appliances may interfere with its function. These sources of interference have been reviewed recently by Edgar Sowton. ${ }^{2}$

The pacing electrode may act as an aerial to transmit electrical signals from the environment to the pacemaker. The consequences of a false signal will depend on its characteristics and on the functional mode of the pacemaker. The most common pacemaker in use at present is the demand type, which paces when the patient's heart rate falls below a predetermined rate but withholds a pacing impulse if a suitable incoming signal denotes a spontaneous QRS complex. External signals with characteristics similar to the endocardial signal may be misinterpreted as coming from the heart and so may inhibit pacing-with potentially serious consequences for the patient. Fixed-rate pacemakers are not affected by environmental sources of interference. Triggered or synchronous pacemakers may respond to external signals by inducing a paced atrial or ventricular tachycardia, which is usually not faster than $170 \mathrm{~min}$. The newer programmable pacemakers may be affected in the same way as the demand units, but in addition the pacemaker setting may be altered by interference signalsphantom programming. ${ }^{3}$ Input filters can reject many unwanted frequencies, but signals similar to those coming from the heart cannot be filtered. Some demand pacemakers identify interference signals and revert to a fixed-rate mode-the "interference rate"; this rate is often faster than the pacing rate and may alert the patient that there is external interference. ${ }^{2}$

The most serious electrical hazards are those that inhibit demand pacemakers with the consequent danger of collapse. Most reported cases have been traced to faults in electric motors in which a capacitor fails to suppress sparking; examples are vacuum cleaners, deep freezers, refrigerators, electric mixers, electric razors, small power tools, and even battery-operated electric razors. The dynamo or alternator on motor cycles, lawn mowers, outboard engines, and even motor cars may inhibit a pacemaker if the patient is within one metre of the engine. Arc welding and fairground "dodgem cars" generate high levels of interference that may cause triggered pacemakers to run at their maximum rate and may inhibit or cause fixed-rate pacing in demand pacemakers. Anti-theft devices that depend on the triggering of an alarm by metal tags attached to goods or on the interruption of a light beam are generally safe and at most inhibit one pacemaker beat. If, however, the patient is close to a detector that is being activated by many people there is a danger of pacemaker inhibition. (In stores with anti-theft devices the patient with a pacemaker may be saved embarrassment by declaring his pacemakerwhich may itself trigger the burglar alarm.) Weapon detectors, contrary to some reports, are unlikely to interfere with pacemakers. Nevertheless, inhibition is a potential danger and patients with a pacemaker should avoid passing through these detectors. Very high-powered radar beams at airport observation areas and defence establishments, at least in theory, inhibit pacemakers but would be unlikely to cause more than the loss of an occasional beat.

Many electrical signals may inhibit one or two pacemaker beats without symptoms; this may occur when a suppressor fails to prevent sparking on electrical equipment such as household or refrigerator thermostats, time switches, and room lighting. Electronic sensor switches and citizens' band radio sets are other sources. Microwave ovens or a small leakage of current from a faulty domestic appliance (such as an electric blanket, kettle, or iron) may switch demand pacemakers to the interference rate, usually without serious consequences for the patient.

Interference with pacemaker function by medical equipment is of particular interest to hospitals and doctors. Surgical diathermy is the best known and most common source of interference, and fatal ventricular fibrillation has been reported. ${ }^{2}$ Diathermy is best avoided, but it may be used cautiously with careful electrocardiographic monitoring provided that the indifferent electrode is well away from the heart or pacemaker site and that the cutting edge of the diathermy is not used near the pacemaker or electrode. The pacemaker is likely to enter the interference rate during diathermy, and sometimes it may be inhibited briefly after diathermy with pacing restarting spontaneously in one or two seconds. Reprogramming of programmable units has also been reported. Short-wave heat treatment may also cause inhibition of pacemakers and is best avoided. Other potential sources of danger are faradic stimulation, transcutaneous stimulators for pain relief, electrical muscle stimulators, low-frequency acupunc- 
ture, and some dental equipment including the ultrasonic cleaner. Investigations into the effect of therapeutic radiation on pacemaker function are conflicting, ${ }^{2}$ and the long-term effect of radiation on electronic components is unknown, but, provided the pacemaker is protected, radiation may safely be given to patients with pacemakers.

The patient faced with this daunting catalogue of potential environmental dangers might well feel insecure. He may be reassured that, though the list is long, the actual danger is, to use Sowton's term, minimal. ${ }^{2}$ Nevertheless, patients with pacemakers should be aware of the more likely sources of interference so that they can learn to avoid them; manufacturers of electrical equipment should recognise the problem and provide warning notices if electrical interference is likely, and pacemaker manufacturers will, no doubt, continue to develop means of filtering and rejecting interference signals.

EOIN O'BRIEN

Consultant Physician (Cardiology),

Charitable Infirmary,

Dublin 1

1 Frank G, Tyers O, Brownlee RR. Power pulse generators, electrodes, and longevity. Prog Cardiovasc Dis $1981 ; 23: 421-34$

2 Sowton E. Environmental hazards for pacemaker patients. $f \mathrm{R}$ Coll Physicians Lond 1982;16:159-64.

${ }^{3}$ Furman S. Spurious pacemaker programming. Pace 1980;3:517-8.

\section{Evoked potentials in neurological diagnosis}

The functional integrity of specific cortical areas and of pathways in the central nervous system can be assessed by recording the electrical potentials associated with specific sensory or motor events. The procedure uses electronic averaging techniques recording from surface electrodes placed over certain areas of the brain or spinal cord. These methods provide a non-invasive objective test which may disclose abnormalities not apparent on clinical examination.

Evoked potentials have now established their place in the diagnostic assessment of patients with several neurological disorders, and their contribution in other disciplines is continuing to be evaluated. ${ }^{1}$ Visual, auditory, and somatosensory evoked potentials have been most widely used diagnostically, while olfactory, cognitive, and movementassociated potentials have yet to find routine application.

The main use of sensory evoked potentials has been in the diagnosis of multiple sclerosis, in which they have proved useful for confirming clinically suspected lesions of the visual, auditory, and somatosensory pathways and-more important -for detecting subclinical lesions. In so doing they may reduce the need for more invasive procedures such as myelography or angiography in some cases. ${ }^{2}$ Most useful has been the pattern reversal visual evoked potential, which is abnormal in $80-90 \%$ of patients with a firm clinical diagnosis of multiple sclerosis with or without previous visual symptoms, ${ }^{34}$ and in $30-50 \%$ of patients with suspected or probable multiple sclerosis. ${ }^{56}$ The characteristic finding is an increase in the latency of the major positive component of the visual evoked potential, which only rarely returns to normal. Small but indistinguishable latency changes may, however, also occur in patients with refractive errors ${ }^{7}$ or other ocular abnormalities, ${ }^{8}$ in pernicious anaemia, ${ }^{9}$ in hereditary ataxias, ${ }^{1011}$ in Charcot-
Marie-Tooth disease, ${ }^{12}$ and in other forms of optic neuropathy. ${ }^{13}$

Auditory brain stem and somatosensory evoked potentials have been less useful than the visual evoked potential, but the combined use of the three techniques provides the highest yield of subclinical abnormalities in patients suspected of suffering from multiple sclerosis. ${ }^{1415}$ Abnormalities of the auditory brain stem potentials have been found in up to $80 \%$ of patients with definite multiple sclerosis ${ }^{15}$ with a previous $\ddot{\vec{F}}$ history or clinical signs of lesions in the brain stem, and in up to $50 \%$ of patients without such signs. ${ }^{1617} \mathrm{~A}$ few patients with isolated optic neuritis are found to have abnormal auditory $\frac{\overline{\bar{N}}}{\bar{D}}$ brain stem potentials pointing to an additional lesion in the brain stem and thereby increasing the likelihood of multiple sclerosis (C Storey, "Role of evoked potentials in the investigation of optic neuritis"; presented at neuro-ophthal- $\vec{\square}$ mology symposium, Melbourne, November 1981). Ab- $\overrightarrow{\vec{\omega}}$ normalities of latency have proved the most useful measures, O especially comparisons of interpeak latency between the two $\frac{0}{3}$ sides. Nevertheless, changes in latency in auditory brain stem i potential components are less definite than in the case of the of visual evoked potential and the abnormalities are in general of more labile; serial studies in patients with multiple sclerosis have shown bidirectional variations. ${ }^{18}$

Abnormal sensory evoked potentials recorded over the scalp or cervical spine, reflecting lesions of the dorsal column/ 은 lemniscal sensory pathway, have been found in over $75 \%$ of $\overrightarrow{-}$ patients with clinically definite multiple sclerosis and in a third $\square$ to a half of patients with probable or suspected multiple sclerosis, including some without sensory symptoms or signs. ${ }^{141920}$ The combined use of upper and lower limb stimulation and the calculation of conduction times in the $\overrightarrow{\vec{\theta}}$ spinal and central portions of the somatosensory pathway has increased the rate of detection of lesions and has helped in locating them. ${ }^{21}$

Abnormalities of evoked potentials may also occur in disorders other than demyelinating diseases, though they are less often of diagnostic value. ${ }^{22} 23$ Of particular importance is the finding of normal evoked potentials in patients with hysterical sensory deficits or malingering. Abnormalities of the visual evoked potential may be found in patients with compressive lesions of the optic nerve or chiasm such as pituitary tumours or meningioma, and the site of compression may be defined more precisely by the use of multichannel recordings and hemifield stimulation. ${ }^{83}$ Characteristic patterns of abnormality are also found in patients with toxic ${ }^{24}$ or hereditary forms of bilateral optic neuropathy..$^{25}$ Auditory brain stem potentials have an important application in assessing auditory

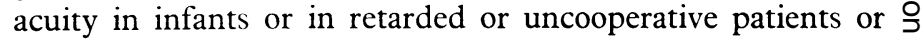
those with psychogenic hearing loss. ${ }^{26}$ They have also proved $\mathrm{N}$ useful in the diagnosis of acoustic neuroma, abnormal responses $\rightarrow$ being found in most cases, ${ }^{27}$ and they may also provide objective evidence of damage to the lower auditory pathway in $\mathbf{N}$ patients with ischaemia or a tumour of the brain stem and in $N$ the postconcussional syndrome. ${ }^{28 * 30}$ They have been used in $\underset{\sim}{\sigma}$ evaluating brain stem function in coma and suspected brain 0 death $^{31}$ but have yet to find general application in these circumstances. Sensory evoked potentials may have a part to play in assessing patients with lesions of the brachial plexus or spinal roots ${ }^{32}$ and in determining the extent of traumatic spinal cord lesions. Evoked potential techniques have also been used to evaluate the severity of cerebral dysfunction after head injury ${ }^{334}$ and in patients with metabolic encephalopathies ${ }^{35} 36$ and coma. ${ }^{37}$

Evoked potentials, therefore, provide a means of objective

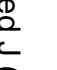

